\title{
A Method of Constructing Robotics Service Platform for Assisting Handicapped or Elderly People
}

\author{
Zuozhong Yin, ${ }^{1,2}$ Dianpeng Wang $\mathbb{D}^{1}{ }^{1}$ and Jihong Liu ${ }^{1}$ \\ ${ }^{1}$ School of Mechanical Engineering \& Automation, Beihang University, Beijing 100191, China \\ ${ }^{2}$ Beijing Research Institute of Automation for Machinery Industry Co. Ltd., Beijing 100120, China \\ Correspondence should be addressed to Dianpeng Wang; wangdianpengemail@163.com
}

Received 7 November 2019; Revised 27 May 2020; Accepted 23 June 2020; Published 15 July 2020

Academic Editor: Gordon R. Pennock

Copyright (C) 2020 Zuozhong Yin et al. This is an open access article distributed under the Creative Commons Attribution License, which permits unrestricted use, distribution, and reproduction in any medium, provided the original work is properly cited.

In order to solve the problem of demand for large capacity storage and high-performance computing resource of intelligent robot navigation and demand-service matching in the field of assisting handicapped or elderly people, overcoming the limitations of carrying resources, the method of constructing robot service platform for assisting handicapped or elderly people (RSP-AHEP) was proposed based on cloud robotics technology. Firstly, the demand of assisting handicapped or elderly people and the corresponding robot service category was analyzed to assure relationship-matching subjects. Secondly, based on VMware and Hadoop cluster technology, the architecture of a three-layer robot service platform was designed, which were universal interface layer, resource service layer, and application layer. Complex computing tasks, such as the matching computing between robot service and demand of the handicapped or elderly people, and the robot path-planning service, were placed in the robot service platform with the advantages of storage and computing resources. Thirdly, the remote communication between the robot and the service platform was realized based on the ROS (Robot Operation System) technology; finally, the function experiments, which included the remote dispatch, path planning, and service response between the service platform and robot, were carried out in the simulation environment. The result verified the feasibility of the proposed method.

\section{Introduction}

The majority of the elderly and disabled groups, for whom robots are designed for serving, are mostly people with limited mobility. Earlier research work focused on robots that met simple needs. And development results are also based on wheelchair-assisted robots [1]. With the development of technology, intelligent robots integrating autonomous navigation, face detection, speech recognition, and other functions have begun to appear, which are used to meet the special and complex task requirements of the elderly and disabled people. At the same time, storing and processing large amounts of information in real time places higher demands on hardware such as mass storage and highperformance computing resources. Due to the limed storage and computing resources carried by the robot itself, it is necessary to invest more time, energy, and financial resources to further improve the learning ability, computing power, and response speed of the service robot to provide better services for the elderly and disability.

The cloud robot technology, which combines cloud computing technology and robotics, provides an effective way to solve the problem that robots have difficulty in further improving their learning and computing ability due to limited onboard resources, and has become a research hotspot in the field of intelligent robots [2]. Among them, in early 2011, the European scientist launched the RoboEarth program, using the Internet to establish an open-source network database for robot learning, which is a possibility for robots around the world to share data and enhance learning ability [3]. Miki Sato designed a general cloud architecture platform from the perspective of people's daily life and conducted various experiments in shopping malls [4]. The results show that the robot has significantly improved in terms of computing power and service response efficiency. It can be seen that cloud robot technology has 
obvious advantages in expanding robot storage space and computing resources, and improving robot learning and computing capabilities.

At present, there are few ways to expand the storage and computing resources of cloud storage technology in the field of helping the elderly and disabled. Therefore, this paper attempts to propose a robot service platform architecture (RSP-AHEP) serving for this field, making full use of storage and computing advantages to achieve the complex service requirements of the elderly and disabled special service needs and robot service matching, robot path planning, the service robot storing and computing resource requirements, and unloading part of the onboard resource burden of the service robot.

\section{Needs of the Elderly and the Handicapped}

Psychologist Abraham H. Maslow proposed that human needs mainly include five levels, namely, physiological needs, security needs, social needs, esteem needs, and selffulfilling needs [5]. Among them, esteem needs and selffulfilling needs are higher requirements based on the first three needs. For older and disabled people, as a special group, their service needs are special in the first three needs mentioned above. Therefore, from the perspective of these users, robotic auxiliary services are also divided into three categories: mobile auxiliary services that meet physiological needs, safety medical services that protect users' safety, and sociality services that meet psychological needs, as shown in Figure 1.

2.1. Mobile Auxiliary Service Needs. According to the analysis of internationally accepted standards, there are 6 indicators, which include eating, dressing, going to bed, going to the toilet, indoor walking, and bathing. If one or two are "cannot do," it is defined as "mild disability". If three to four are "cannot do," it is defined as "moderate disability". If three to four are "cannot do," it is defined as "moderate disability". While five to six items are "cannot do", it is defined as "severe disability" [6]. The indicators are sorted according to the disability status and the specific indicators, as shown in Table 1.

Elderly and disabled people have different degrees of degeneration in terms of bodily functions. Among them, the larger demand for robotic auxiliary functions is mobile assistance. Helping the elderly and disabled people to participate in their own activities can not only enhance their self-care ability but also help them to increase their pursuit of other aspects of life and fun. The correspondence between disability status and robot service-demand configuration is shown in Table 2.

2.2. Safety Medical Service Needs. Taking into account the poor health of the elderly and disabled people, unstable health conditions, inconvenient movements, etc., services such as emergency medical care and safety monitoring are set up in the service platform. By detecting the physiological parameters of the user in real time, the user's health status is

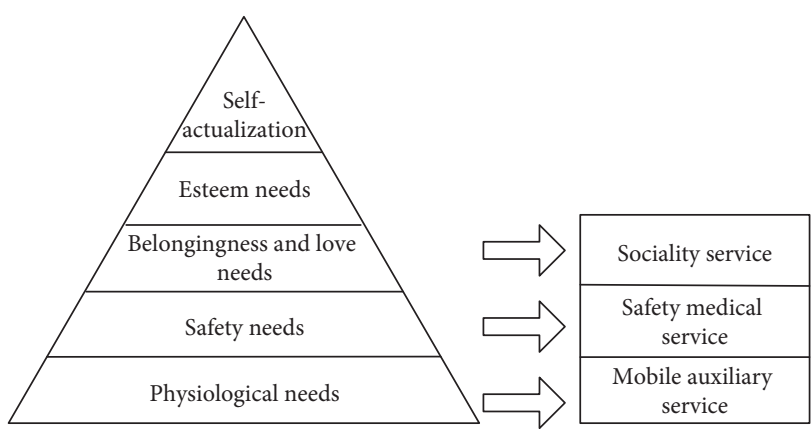

FIgURE 1: Map between needs and service.

TABLE 1: Correspondence between indicators and disability.

\begin{tabular}{lccc}
\hline Indicators & $\begin{array}{c}\text { Mild } \\
\text { disability }\end{array}$ & $\begin{array}{c}\text { Moderate } \\
\text { disability }\end{array}$ & $\begin{array}{c}\text { Severe } \\
\text { disability }\end{array}$ \\
\hline Bathing & Cannot & Cannot & Cannot \\
Indoor & Cannot & Cannot & Cannot \\
walking & Ok & Cannot & Cannot \\
Going to toilet & Ok & Cannot & Cannot \\
Going to bed & Ok & Ok & Cannot \\
Dressing & Ok & Ok & Cannot \\
Eating & & &
\end{tabular}

TABle 2: Correspondence between disability and needs of robot service type requirement.

\begin{tabular}{lc}
\hline Disability & Robot service type \\
\hline $\begin{array}{l}\text { Mild disability } \\
\text { Moderate disability }\end{array}$ & Walkers \\
Severe disability & Walkers, rehabilitation robot \\
\hline
\end{tabular}

evaluated, and the user is provided with timely and accurate medical services.

2.3. Social Service Needs. Compared with the young, the elderly and the disabled have more time for solitude and more demand for spiritual services. The spiritual needs, including the need for personal respect, emotional support, and psychological comfort, are important elements in helping the elderly. Providing social function services for the elderly is an important part of improving the quality of life of the elderly.

\section{Robot Service Platform Architecture for the Field of Helping the Elderly}

Currently, cloud robot architectures include the ad hoc cloud (machine-to-machine communication, M2M) and cloud (machine-to-cloud communication, M2C) architectures [2], and the Ubiquitous Network Robot Platform (UNR-PF, a platform for cloud networked robotic services) [4] architecture. The former architecture discusses the allocation and communication of computing resources between the robot and the cloud. The latter architecture introduces a unified platform for different service 
applications. These studies have laid the technical foundation for the promotion and application of cloud robots. In combination with the service needs of the elderly and the disabled and the cloud service platform construction technology $[7,8]$, the RSPAHEP robot service platform architecture for the elderly and disabled people is proposed. RSP-AHEP is a service-demand matching platform based on service-oriented architecture (SOA) [9] and cloud computing technology [10]. The overall architecture of the service platform is shown in Figure 2. The RSPAHEP architecture is a service-oriented hierarchical architecture. It consists of three layers: the universal interface layer, the resource service layer, and the application layer, as shown by the dotted line in Figure 2.

3.1. Platform Maintainer/Robots/Handicap. Platform maintainer is responsible for monitoring and maintaining the RSP-AHEP daily operation. Robots with sensors could complete instructions from the RSP-AHEP. Handicap means user ends for uploading user's service requirements.

3.2. Universal Interface Layer. Data management and unified access interface for the resource service layer are implemented by Hadoop Distributed File System (HDFS, a distributed file system that provides high-throughput access to application data) and the parallel computing model MapReduce based on VMware software and the Apache Hadoop project (opensource software for reliable, scalable, distributed computing) [11]. Message interaction is achieved by the ROS-based publish/subscribe node and the TCP/IP communication protocol between the service platform and the external [12].

3.3. Source and Service Layer. The resource service layer includes two parts: resource storage and demand-service matching computing. The stored resources include robot state information, function information and service platform storage, and computing resource occupancy information. The demand-service matching computing part is used to match robot resource and the user requirements, which are descripted in Section 2. In the overall task planning, task decomposition service is used to decompose a task which is from the application layer. Then, task allocation service [13] completes matching computing between the decomposed tasks and the robot resource. Finally, the matching result is transmitted to the application layer.

Therefore, it contains the following function: (1) user need and robot resource data update and storage; (2) dynamic matching between user requirements communicated and robot resources; (3) result transmission including the internal matching result fed back to the application layer and the robot instruction being output to the robot.

This layer of the RSP-AHEP could process and store more information which processed on the robot in previous usages [1].

3.4. Application Layer. The application layer includes two functional modules: user application module and platform maintainer monitoring and maintenance module. Under the user application module, the user accesses the service center resources and the corresponding service through the website, the human-computer interaction interface (mobile terminal, PC terminal, dedicated terminal, etc.). The application layer completes service requirements parsing and result transmission. With the monitoring and maintenance module, the platform maintainer performs resource allocation monitoring and system maintenance through the backstage of the service platform to ensure the stable operation of the service system.

The service options are set based on the content of Section 2, which include mobile auxiliary service, safety medical services, and sociality services.

\section{Workflow of Service Platform in Nursing Scene}

The nursing home is a social welfare organization that provides services for the elderly. It is a typical applying scene of robots designed for the elderly and disabled. Taking RSPAHEP as the example of user-cleaning request in this service scenario, the entire service response information flow is shown, as shown in Figure 3.

\subsection{Submitting Service Request}

(1) When the user has the room-cleaning service requirement, the handicap submits the service request to the cloud platform through Internet protocol [12] with multiple input recognition modes such as touch and voice, as shown in instruction 1(cleaning requirement)

(2) After the service platform application layer parses the service requirement content (cleaning requirement) from instruction 2 (transmitted from the universal interface layer), the parsed requirement is submitted to the resource service layer, as shown in instruction 3

4.2. Processing Service Request. The resource service layer contains available robot resources and matching rules between resource 5 (the cleaning robot) and demand 4 (cleaning requirement).

(1) The resource service layer matches robot resources with user request in the platform by querying the available robot resource 7 . If the match is successful, the layer sends the matching result (cleaning robot ID and serving time) to the application layer, as shown in instruction 8 .

(2) The application layer sends the matching result information to the user through the ROS_bridge transmission protocol [12], as shown in instructions 9 and 10.

(3) The resource service layer sends the service command (goal position and path information) to the cleaning robot through the universal interface layer, as shown in instructions 11 and 12 . 


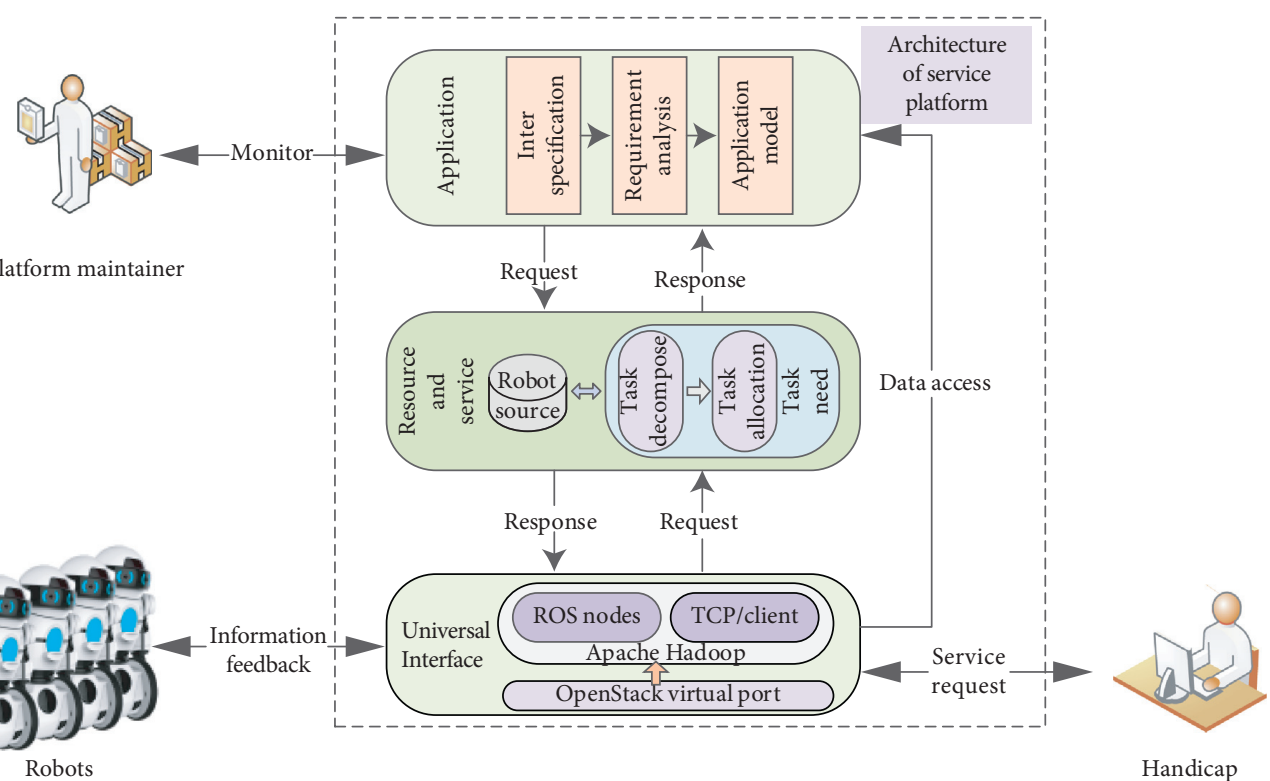

FIGURE 2: RSP-AHEP: robotics service platform for assisting handicapped or elderly people.

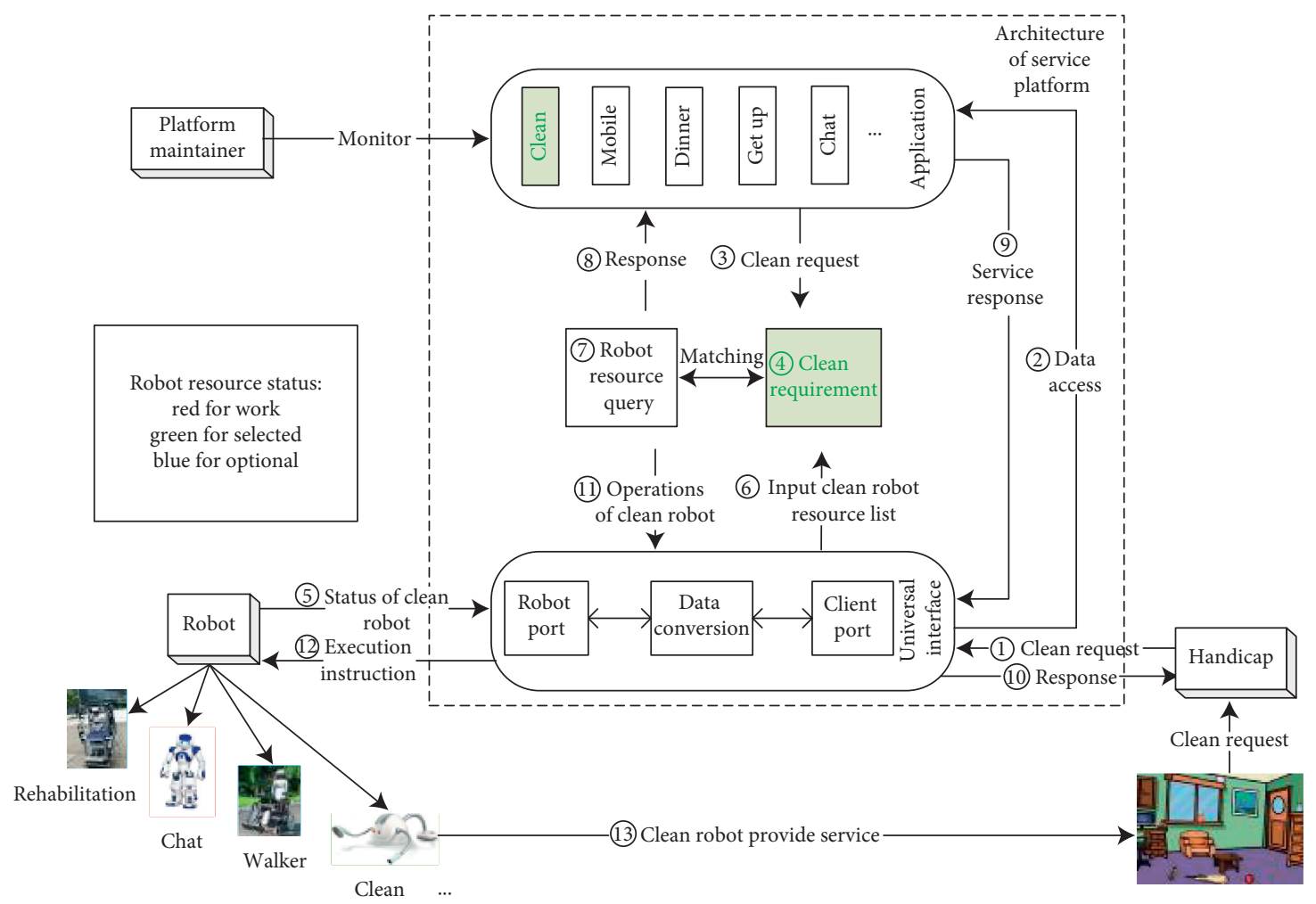

FIGURE 3: The workflow case of response for clean request in RSP-AHEP.

4.3. Responding Service Request. When receiving instructions from the service platform, the cleaning robot moves to the designated location to execute the task according to the path-planning information provided by the service platform. After completing the task, the robot automatically moves back to the specified position, as shown in instruction 13, shown in Figure 3.

\section{Verification and Discussion}

Two different tasks are designed for testing the service response of the RSP-AHEP:

Task 1: assuming that the user submits the assisting eating request through voice input indoors, it is 
designed for testing the remote deployment and pathplanning function of the RSP-AHEP

Task 2: supposing the health physician needs to get daily physiological parameters of two patients, who lived in different rooms, it is designed for testing taskplanning and robot status-monitoring function of the RSP-AHEP

The service platform is a Hadoop cluster in a multivirtual machine environment based on the VMware software platform, and the communication adopts a public/listen node form of a ROS (Robot Operation System). The hardware configuration is as follows: the server platform adopting the Intel(R) Core(TM) i5-3470 processor 4G RAM memory and the service robot adopting the TurtleBot-robot in the simulation environment, using a microphone as a voice handicap input device.

In Task 1, the vocabulary of the ROS pocketsphinx package $[12,14]$ is stored in the RSP-AHEP, which the new voice instruction information is defined based on. When the RSP-AHEP received the voice signal from the user, the voice_recog.py node in the ROS recognized the voice information as the defined instruction ID in the pound-etsphinx. Then, information, which included the status and coordinate of request, was extracted from an instruction ID array. After that, RSP-AHEP detected the status of all robot resource for assisting eating based on the request. When detecting the available food service robot, coordinate information of robot and user were posted to the ros_navigation node. RSP-AHEP completed path planning and sent the instruction to ros_navigation node for ensuring that the service robot reached the specified coordinate position. The test environment map was generated by scanning a room with obstacles by the ros_mapping package, as shown in Figure 4.

As shown in Figure 5, after receiving the instruction, the service robot starts from the initial position point $A$, bypassed the obstacle through the path planning and reached the target point $\mathrm{B}$. It was realized remote scheduling of service robots by RSP-AHEP.

In Task 2, the environment map is the ROS selfcontained map. The initial position of the detection service robot is the arrow position. Through the RSPAHEP path planning, the robot sequentially reaches the mission target point $\mathrm{T} 1$ and the mission target point $\mathrm{T} 2$, as shown in Figures 6 and 7. The planning path was marked by a green thin line as shown in Figures 6 and 7 . As Figure 8 shows, the rviz (node label) recorded the state information log of the robot, which first came to T1 at \#1 time (stamp label) and then to T2 at \#2 time (stamp label).

It can be seen from the above two tests that the proposed method of RSP-AHEP realized path planning, remote deployment, and remote service response functions for the service robot.

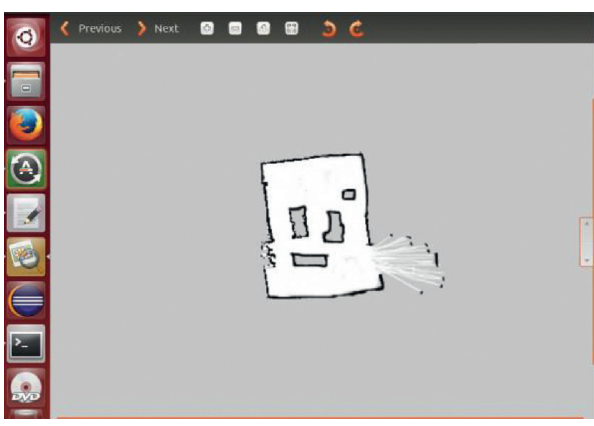

FIgURE 4: Environment map.

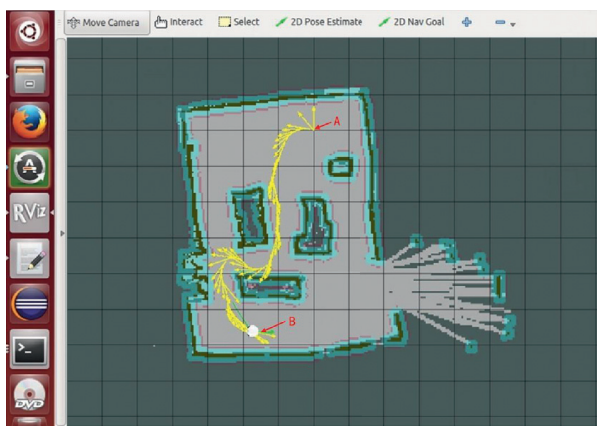

FIGURE 5: Response of the service robot for voice request.

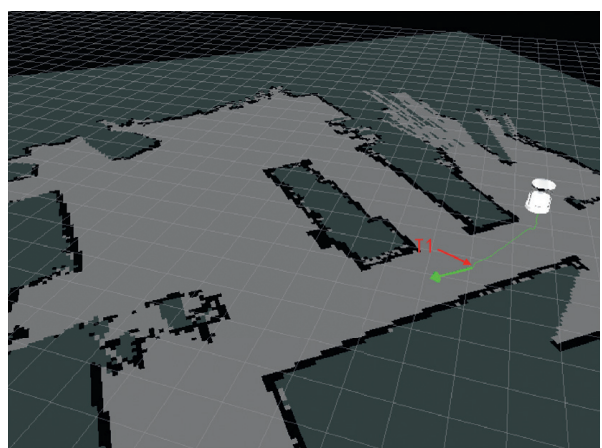

FIgURE 6: Path-planning response of T1 in RSP-AHEP.

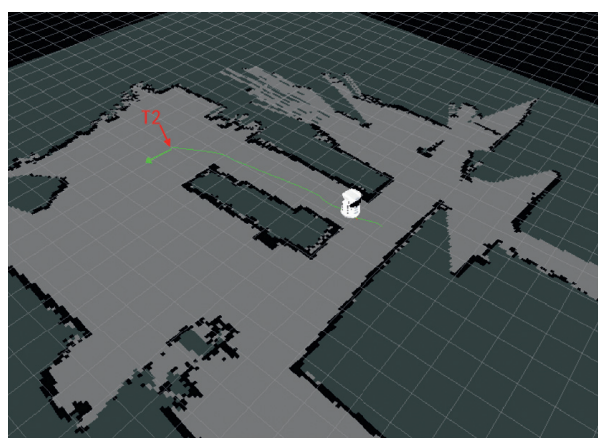

Figure 7: Path-planning response of T2 in RSP-AHEP. 


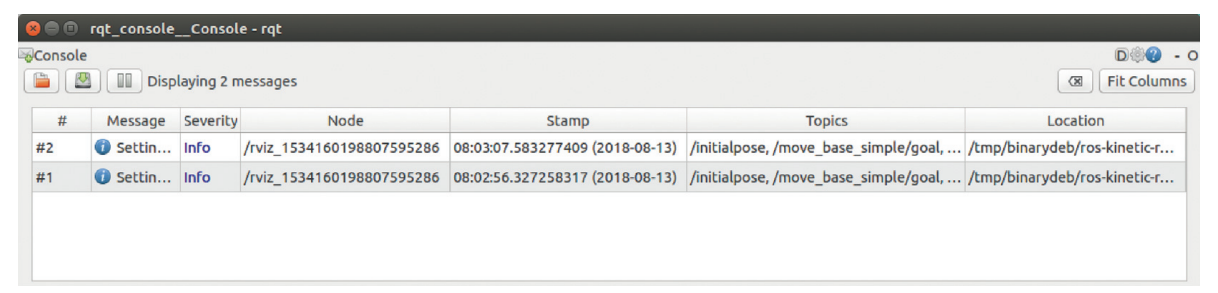

Figure 8: Response log of the service robot for voice request.

\section{Conclusion}

Combining cloud robot technology with the needs of helping the elderly and disabled, a method was provided to solve the limited storage space resources of the service robot. And a feasible solution was also proposed for constructing a unified regulatory management service robot resource platform. Furthermore, a workflow case in nursing scene showed feasibility of functional modules, such as remote scheduling, path planning, and service response, in RSP-AHEP. Although the research results of cloud robot technology in the specific application level of helping the elderly and helping the disabled are still relatively preliminary, with the rapid development of related technologies such as cloud computing, there are huge potentials in solving the traditional bottlenecks in the field of helping the elderly and handicapped, such as task planning of heterogeneous robots and multiservice robot collaboration for assisting the disability.

\section{Data Availability}

For the manuscript, (1) the vocabulary of the ROS pocketsphinx package contains lots of vocabulary, which is used to obtain text instructions from the voice information. The vocabulary of the ROS pock-etsphinx package data used to support the findings of this study are included within the article. (2) The test 1_nature_data contains coordinates of robot localization and task position, which are extracted from our own SLAM map. The test 1_nature_data data used to support the findings of this study are included within the supplementary information file. (3) The test 2 is based on the ROS self-contained map, which can be accessed from the following site. The test 2 data used to support the findings of this study can be accessed from https://github.com/ros-visualization/rviz.

\section{Conflicts of Interest}

The authors have no conflicts of interest.

\section{Acknowledgments}

This work was supported by the National Key R\&D Program (no. 2016YFF0202003).

\section{References}

[1] I. P. Ktistakis, N. G. Bourbakis, and N. G. Bourbakis, "Assistive intelligent robotic wheelchairs," IEEE Potentials, vol. 36, no. 1, pp. 10-13, 2017.

[2] H. Zhang and L. Zhang, "Cloud robotics architecture: trends and challenges," in Proceedings of 2019 IEEE International Conference on Service-Oriented System Engineering (SOSE), pp. 362-3625, San Francisco East Bay, CA, USA, April 2019.

[3] H. Du, W. Xu, B. Yao, Z. Zhou, and Y. Hu, "Collaborative optimization of service scheduling for industrial cloud robotics based on knowledge sharing," Procedia CIRP, vol. 83, pp. 132-138, 2019.

[4] J. Liu, F. Zhou, L. Yin, and Y. Wang, "A novel cloud platform for service robots," IEEE Access, vol. 7, pp. 182951-182961, 2019.

[5] K. Healy, "A theory of human motivation by Abraham H. Maslow (1942)," British Journal of Psychiatry, vol. 208, no. 4, p. 313, 2016.

[6] A. L. Danielewicz, J. C. dos Anjos, J. L. Bastos, A. C. Boing, and A. F. Boing, "Association between socioeconomic and physical/built neighborhoods and disability: a systematic review," Preventive Medicine, vol. 99, pp. 118-127, 2017.

[7] A. Vakili and N. J. Navimipour, "Comprehensive and systematic review of the service composition mechanisms in the cloud environments," Journal of Network and Computer Applications, vol. 81, pp. 24-36, 2017.

[8] Y. Zhang, G. Zhang, Y. Liu, and D. Hu, "Research on services encapsulation and virtualization access model of machine for cloud manufacturing," Journal of Intelligent Manufacturing, vol. 28, no. 5, pp. 1109-1123, 2017.

[9] W. Tan, Y. Fan, A. Ghoneim, M. A. Hossain, and S. Dustdar, "From the service-oriented architecture to the web API economy," IEEE Internet Computing, vol. 20, no. 4, pp. 64-68, 2016.

[10] E. Jonas et al., "Cloud programming simplified: a berkeley view on serverless computing," 2019, http://arxiv.org/abs/ 1902.03383 .

[11] Rodrigues and R. Alceu, "Integrating NoSQL, relational database, and the hadoop ecosystem in an interdisciplinary project involving big data and credit card transactions," Information Technology-New Generations, pp. 443-451, Springer, Cham, Switzerland, 2018.

[12] http://www.willowgarage.com/.

[13] X. Kong, Y. Gao, T. Wang, J. Liu, and W. Xu, "Multi-robot task allocation strategy based on particle swarm optimization and greedy algorithm," Proceedings of 2019 IEEE 8th Joint International Information Technology and Artificial Intelligence Conference (ITAIC), Chongqing, China, IEEE, May 2019.

[14] G. Toffetti and T. M. Bohnert, "Cloud robotics with ROS," in Robot Operating System (ROS). Studies in Computational Intelligence, A. Koubaa, Ed., Vol. 831, Springer, Cham, Switzerland, 2020. 